\title{
Self-consistent modelling of X-ray photoelectron spectra from air-exposed polycrystalline TiN thin films
}

\author{
Grzegorz Greczynski and Lars Hultman \\ Journal Article
}

\section{Tweet}

N.B.: When citing this work, cite the original article.

Original Publication:

Grzegorz Greczynski and Lars Hultman, Self-consistent modelling of X-ray photoelectron spectra from air-exposed polycrystalline TiN thin films, Applied Surface Science, 2016. 387(), pp.294-300.

http://dx.doi.org/10.1016/j.apsusc.2016.06.012

Copyright: Elsevier

http://www.elsevier.com/

Postprint available at: Linköping University Electronic Press

http://urn.kb.se/resolve?urn=urn:nbn:se:liu:diva-132466

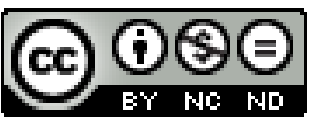




\title{
Self-consistent modelling of X-ray photoelectron spectra from air-exposed polycrystalline TiN thin films
}

\author{
G. Greczynski and L. Hultman \\ Thin Film Physics Division, Department of Physics (IFM), Linköping University, \\ SE-581 83 Linköping, Sweden
}

\begin{abstract}
We present first self-consistent modelling of x-ray photoelectron spectroscopy (XPS) Ti 2p, N 1s, O 1s, and C 1s core level spectra with a cross-peak quantitative agreement for a series of TiN thin films grown by dc magnetron sputtering and oxidized to different extent by varying the venting temperature $T_{v}$ of the vacuum chamber before removing the deposited samples. Soobtained film series constitute a model case for XPS application studies, where certain degree of atmosphere exposure during sample transfer to the XPS instrument is unavoidable. The challenge is to extract information about surface chemistry without invoking destructive precleaning with noble gas ions. All TiN surfaces are thus analyzed in the as-received state by XPS using monochromatic Al Ka radiation $(\mathrm{h} v=1486.6 \mathrm{eV})$. Details of line shapes and relative peak areas obtained from deconvolution of the reference Ti 2p and $\mathrm{N} 1$ s spectra representative of a native TiN surface serve as an input to model complex core level signals from air-exposed surfaces, where contributions from oxides and oxynitrides make the task very challenging considering the influence of the whole deposition process at hand. The essential part of the presented approach is that the deconvolution process is not only guided by the comparison to the reference binding energy values that often show large spread, but in order to increase reliability of the extracted chemical information the requirement for both qualitative and quantitative self-consistency between component peaks belonging to the same chemical species is imposed across all core-level spectra (including often neglected $\mathrm{O}$ 1s and $\mathrm{C} 1 \mathrm{~s}$ signals). The relative ratios between contributions from different chemical species vary as a function of $T_{v}$ presenting a self-consistency check for our model. We propose that the cross-
\end{abstract}


peak self-consistency should be a prerequisite for reliable XPS peak modelling as it enhances credibility of obtained chemical information, while relying entirely on reference binding energy values introduces large ambiguity.

Keywords: TiN, $\mathrm{TiO}_{2}$, oxynitride, XPS, magnetron sputtering, peak fitting, chemical states

\section{Introduction}

X-ray photoelectron spectroscopy (XPS) is commonly employed to study surface chemistry and composition of various compounds in bulk or thin film form. A model example is TiN with related ternary thin film alloys grown by magnetron sputter deposition, that attract tremendous scientific and technological interest due to their unique combination of properties including high electrical conductivity, ${ }^{1,2}$ good high-temperature oxidation resistance, ${ }^{3,4}$ high hardness, ${ }^{5,6}$ and tunable optical absorption, all of which make them very attractive for applications as wear-resistant coatings on cutting tools ${ }^{7,8}$ and engine components, ${ }^{9,10}$ as well as diffusion barriers in electronic devices. ${ }^{11,12}$ Large attention has thus been and continues to be devoted to the spectroscopic studies of both in-situ and ex-situ grown $\mathrm{TiN}_{x}$ films with $x$ varying from 0 to 1 and the nature of $\mathrm{Ti} 2 \mathrm{p}$ satellite peaks that result from nitridation. ${ }^{13,14,15,16,17,18,19,20}$ The TiN surface oxidation studies performed under wellcontrolled conditions also earned attention due to direct consequences for applications. ${ }^{21,22,23}$ In these reports, almost exclusively, Ti 2p and N 1s core-level spectra are in focus. Due to high complexity involved particularly in the case of $\mathrm{Ti} 2 \mathrm{p}$ spectra, which in addition to main peaks shows also satellite loss features, ${ }^{13}$ there is no consensus as to how the unambiguous spectra deconvolution should be performed. Typically, after background subtraction, spectra is fitted with a number of component peaks to obtain satisfactory fit and peak assignment is based 
entirely on the apparent binding energy (BE) values by comparison to the existing literature. The chemical information extracted in such way suffers, however, from two essential problems. First, the reported reference BE values for given chemical state exhibit large spread, often on the same order as the BE difference between two different chemical states. For example, NIST data base ${ }^{24}$ contains 91 entries for $\mathrm{TiO}_{2}$ with the $\mathrm{Ti} 2 \mathrm{p}_{3 / 2} \mathrm{BE}$ varying from 458.0 to $459.60 \mathrm{eV}$ and $\mathrm{O}$ 1s from 529.4 to $531.2 \mathrm{eV}$, which clearly prevents unambiguous assignment of observed spectral features. This situation is illustrated in Figure 1 for the case of $\mathrm{Ti} 2 \mathrm{p}_{3 / 2}$ core-level and the chemical states relevant to this work..$^{24,25,26,27}$ The overlap between BE range assigned to different chemical species is evident, which often results in an arbitrary spectra interpretation and contradicting results.

Second problem with the commonly applied deconvolution approach is that such procedure neglects prerequisites of qualitative, and even more so quantitative, self-consistency across all core level signals. That is, the presence of component $A 1$ in the deconvoluted spectrum of element $A$ assigned to $A_{m} B_{n}$ formation requires that the corresponding $B 1$ component peak is present in the core-level signal of element $B$ (qualitative self-consistency). Moreover, the elemental concentrations extracted from $A 1$ and $B 1$ peak areas should reflect the compound stoichiometry $m / n$ (quantitative self-consistency). To our best knowledge, such self-consistent peak models of TiN XPS spectra have not been presented before.

In addition, vast majority of available XPS literature devoted to TiN oxidation studies neglects presentation and discussion of $\mathrm{O} 1 \mathrm{~s}$ and $\mathrm{C} 1$ s spectra, which, in our opinion, constitutes an inherent part of the consistent peak model. Unintentional oxygen and carbon contamination in the analyzed surface region is difficult to avoid, not only during sample transfer to the XPS instrument, but also during the film growth with commercial purity targets and gases under high vacuum conditions. High TiN reactivity towards oxygen accounts for surface oxidation detected by XPS even in the films grown in situ in the ultra-high-vacuum (UHV) 
environment. ${ }^{28}$ The situation is not entirely resolved by often used noble-gas ion etching performed prior to XPS analyses, which leads to atomic mixing, forward sputtering, and recoil implantation of surface impurities into deeper layers, apart from the fact that the whole process affects structure and composition in the surface region to depths comparable to XPS probing length.

To address issues outlined above, we present a complete self-consistent XPS core-level peak model for a set of polycrystalline TiN films grown by magnetron sputtering and oxidized to various extent by varying the temperature during following air exposure (venting temperature, $T_{v}$ ). All core level spectra exhibit pronounced changes as a function of $T_{v}$, which makes them ideal candidates for testing the self-consistency of our peak models. Spectra deconvolution is performed in the step-by-step manner starting with the simplest case of a reference native TiN surface, which then serves as an input to more complex peak models of air-exposed TiN films with oxides and oxynitrides contributions. For each TiN layer, not only the qualitative, but also the quantitative self-consistency between component peaks belonging to the same chemical species is obtained across all core-level spectra including often neglected $\mathrm{O}$ 1s and C 1s signals. This approach drastically enhances reliability of the extracted chemical information that is obtained in a non-destructive way.

\section{Experimental}

\subsection{Film growth}

Polycrystalline TiN films are grown by reactive dc magnetron sputtering (DCMS) in a CC800/9 CemeCon AG system using rectangular $8.8 \times 50 \mathrm{~cm}^{2}$ target and Ar: $\mathrm{N}_{2}$ (4:1) gas mixture. $\mathrm{Si}(001)$ substrates previously cleaned in aceton and isopropanol are biased at $-60 \mathrm{~V}$ and mounted $6 \mathrm{~cm}$ away from the target surface. The system base pressure $p_{b}$ is $2.3 \times 10^{-6}$ Torr $(0.3 \mathrm{mPa})$ while the total pressure during deposition is $3 \mathrm{mTorr}(0.4 \mathrm{~Pa})$. The average target 
power is $4 \mathrm{~kW}$ and the resulting film thickness is $1.2 \mu \mathrm{m}$. The heating is accomplished with two resistive heaters operating at $8.8 \mathrm{~kW}$ each, ensuring the substrate temperature $T_{s}$ of 430 ${ }^{\circ} \mathrm{C}$.

Following the deposition, TiN films are exposed to atmosphere at different venting temperatures $T_{v}$, from $T_{v}=T_{s}=430{ }^{\circ} \mathrm{C}$ (an immediate vent), to $T_{v}=29^{\circ} \mathrm{C}$ (after $840 \mathrm{~min}$ long wait in vacuum to allow for complete cool down). All samples are subsequently transferred to the load-lock chamber of the UHV XPS system. As recently demonstrated this procedure results in a various degree of surface oxidation, depending on $T_{v}{ }^{29}$

To obtain the reference $\mathrm{Ti} 2 \mathrm{p}$ and $\mathrm{N}$ 1s spectra from native TiN surfaces while avoiding detrimental effects of ion sputtering, additional TiN film is deposited and in-situ capped with 1.5-nm-thick $\mathrm{Al}$ layers (referred to as $\mathrm{Al} / \mathrm{TiN}$ ) in the deposition system prior to air-exposure and loading into the XPS instrument. ${ }^{30}$

\subsection{Film characterization}

$\theta-2 \theta$ x-ray diffraction scans indicate that TiN films are single-phase NaCl-crystal structure and possess 111 preferred orientation. The N/Ti ratio assessed by Rutherford backscattering spectroscopy (RBS) is $1 \pm 0.01$, while time-of-flight energy elastic recoil detection analyses (ToF-E ERDA) ${ }^{31}$ give $\mathrm{C}$ and $\mathrm{O}$ bulk concentrations of 0.4 and 0.2 at\%, respectively.

Ti 2p, N 1s, O 1s, and C 1s core-level XPS spectra are obtained from TiN and Al/TiN films in an Axis Ultra DLD instrument from Kratos Analytical (UK). The base pressure in the system during spectra acquisition is $1.1 \times 10^{-9}$ Torr $\left(1.5 \times 10^{-7} \mathrm{~Pa}\right)$. Monochromatic Al Ka radiation $(\mathrm{hv}=1486.6 \mathrm{eV})$ is used and the anode power is set to $225 \mathrm{~W}$. All spectra are collected from the area of $0.3 \times 0.7 \mathrm{~mm}^{2}$ and at normal emission angle. Spectrometer BE scale is calibrated to the Fermi energy cut-off of the sputter-cleaned polycrystalline Ag film, resulting 
in $\mathrm{Ag} 3 \mathrm{~d}_{5 / 2}$ peak position of $368.3 \mathrm{eV}$. The analyzer pass energy is set to $20 \mathrm{eV}$ which yields the full width at half maximum of $0.55 \mathrm{eV}$ for the $\mathrm{Ag} 3 \mathrm{~d}_{5 / 2}$ peak. Spectra deconvolution and quantification is performed using CasaXPS software package and sensitivity factors supplied by instrument manufacturer. ${ }^{32}$

\section{Results and Discussion}

\subsection{Binding energy reference}

We record for each sample the highest kinetic energy portion of the valence band spectra around Fermi energy, and define the BE scale reference by setting the Fermi level cutoff energy as " 0 ". This is done in order to avoid uncertainty resulting from the fact that $\mathrm{C} 1 \mathrm{~s}$ $\mathrm{BE}$ depends on the type of surface oxides formed during venting procedure ${ }^{29}$ rather than following a common approach of referencing to C 1s line. Such procedure results in Ti 2p $3 / 2$ peak from native TiN surface at $455.03 \mathrm{eV}$, which falls well within the BE range of previously reported values (see Fig. 1). The BE of C 1s peak corresponding to C-C/C-H component of the adventitious carbon varies however, from $284.04 \mathrm{eV}$ with the $T_{V}=330{ }^{\circ} \mathrm{C}$ sample to 284.68 $\mathrm{eV}$ in the case of $T_{v}=430{ }^{\circ} \mathrm{C}$ TiN film, see Table 1.

\subsection{Peak model construction}

In order to deconvolute very complex core-level spectra obtained from air-exposed TiN surfaces, primarily Ti 2p, we apply a step-by-step procedure starting with the simplest case of the native TiN (Al-capped) sample, where only two Ti 2p $3 / 2$ components are present (main TiN peak and the satellite, TiN-sat, see Fig. 2(a)). Line shapes, 2 $\mathrm{p}_{3 / 2}-2 \mathrm{p}_{1 / 2} \mathrm{BE}$ splitting, and the $2 \mathrm{p}_{3 / 2} / 2 \mathrm{p}_{1 / 2}$ area ratio obtained for pairs of TiN and TiN-sat peaks are then constrained in modelling of more complex spectra with up to four contributions. In addition, the BE difference between TiN and TiN-sat peaks and the relative TiN/TiN-sat peak areas are also fixed at values determined from $\mathrm{Al} / \mathrm{TiN}$ sample. These constraints in peak modeling are necessary to inforce 
mathematical least squares solutions that are physically meaningful. The fitting parameters include Ti 2p $3 / 2$ peak area, full-width-at-half-maximum (FWHM), and peak position. For all peak models, Shirley background ${ }^{33}$ subtraction is used while line shapes include Voigt functions (denoted as "GL" in Table 1). For correct representation of TiN component in the Ti 2p spectra, asymmetrical line shapes are employed in the form of Voigt functions with tail modifiers. ${ }^{34}$ An additional global constraint to the model is the fact that the particular line shape representing given chemical state is the same for all samples.

In the spirit of following the Ockham's rule, the number of new component peaks is kept to the minimum yielding satisfactory fit. Also, in each case, every component peak has a well-defined physical interpretation.

Essential to the modelling of XPS core level spectra presented here is that not only qualitative, but also quantitative cross-peak self-consistency is achieved. That is, the presence of component $A 1$ in the deconvoluted spectrum of element $A$ assigned to $A_{m} B_{n}$ formation requires that the corresponding $B 1$ component peak is present in the core-level signal of element $B$ (qualitative self-consistency). The elemental concentrations extracted from $A 1$ and B1 peak areas should also reflect the compound stoichiometry $\mathrm{m} / \mathrm{n}$ (quantitative selfconsistency).

\subsection{Native TiN}

Vast majority of XPS analyses on TM nitride films is performed following the in situ sputter cleaning in order to remove surface contaminations and native oxide layers. Inert gas ions (typically $\mathrm{Ar}^{+}$) with the energy of a few $\mathrm{keV}$ used for this purpose lead, however, to changes in the near-surface sample region due to preferential sputtering of lighter atoms, forward recoil implantation, surface roughening, etc, all of which make quantitative, as well as, qualitative chemical analyses extremely challenging. This is a particular problem for 
metal/gas compounds and alloys such as nitrides and oxides, where the mass difference between film constituents is large (e.g., for TiN $m_{T i}=3.42 \times m_{N}$ ).

The thickness of the surface layer modified by $\mathrm{Ar}^{+}$ion bombardment is a function of ion energy $E_{\mathrm{Ar}^{+}}$and the ion incidence angle $\psi$, and corresponds to the average Ti and $\mathrm{N}$ primary recoil projected range $\xi$, which can be estimated from Monte-Carlo based TRIM (Transport of Ions in Matter) ${ }^{35}$ simulations of ion/surface interactions. With very gentle conditions, $E_{A r^{+}}$ $=0.5 \mathrm{keV}$ and $\psi=70^{\circ}, \xi_{T i}=12 \AA$ and $\xi_{N}=6 \AA$ for Ti and $\mathrm{N}$ recoils, respectively. This is comparable to typical XPS probing depths that are controlled by electron inelastic mean free paths $\lambda$. The fraction of the XPS signal intensity $I_{d}$ originating from a surface layer of thickness $d$ is given by $[1-\exp (-d / \lambda)]$. With $\lambda=18 \AA$ (for electrons originating from Ti 2p core levels with kinetic energy of $\sim 1030 \mathrm{eV}$ ) [Ref.36] and $d=\xi_{T i}$, the percentage contribution to the Ti 2p XPS signal originating from the $\mathrm{Ar}^{+}$ion beam modified surface layer is $\sim 60 \%$. This simple estimate indicates that even very mild ion etching conditions may have serious influence on the XPS spectra, as for artifact-generation. For more commonly used ion etching parameters, $E_{\mathrm{Ar}^{+}}=4 \mathrm{keV}$ and $\psi=45^{\circ}$, the contribution from ion-modified layer is as much as 90\%, making chemical bonding analysis of the studied as-deposited material a futile exercise.

In order to circumvent these obstacles we recently showed that $\mathrm{Al}$ capping layers with thickness lower than $2 \mathrm{~nm}$ can be successfully used to prevent oxidation of magnetronsputtered polycrystalline TiN film surfaces during air exposure prior to insertion into the UHV XPS system. ${ }^{30}$ This allowed for non-destructive acquisition of high-resolution core-level spectra representative of clean samples, essentially identical to those obtained from singlecrystal TiN/MgO(001) films grown and analyzed in-situ in a UHV XPS system. In addition, N/Ti ratio obtained from the Al-capped TiN samples was in a very good agreement with RBS and ToF-ERDA results, and significantly higher than the value obtained from ion-etched surfaces in which case severe $\mathrm{N}$ loss resulting from preferential resputtering prevents 
meaningful quantitative analyses. The XPS-determined N/Ti values for the mild ion etching with relatively low $\mathrm{Ar}^{+}$energy of $0.5 \mathrm{keV}$ and shallow incidence angle $\psi=70^{\circ}$ result in N/Ti $=0.74 \pm 0.03$, which should be compared to $0.98 \pm 0.03$ obtained by XPS for TiN films with Alcap layer, and the bulk concentration of 0.96 \pm 0.01 determined by RBS and ToF-ERDA. Still, the surface composition probed by XPS may deviate from the bulk's for thermodynamical and/or synthesis processing reasons. Thus, the need to develop more advanced modelling methods is further motivated.

Ti 2p and N 1s spectra of TiN surfaces obtained from Al-capped samples are shown in Figs. 2(a) and 3(a). The detail analyses of Ti 2p, $\mathrm{N}$ 1s, and $\mathrm{O}$ 1s signals reveal that, to the XPS detection limits, surfaces are free from oxygen contamination. Both Ti $2 p$ and $\mathrm{N}$ 1s spectra shown in Figs. 2(a) and 3(a) are identical to those obtained from the in-situ grown single-crystal TiN/MgO(001) films. ${ }^{37}$ In particular, the intensities of Ti 2p satellite peaks, discussed in detail below, are the same, which excludes a significant contribution due to either $\mathrm{TiO}_{x} \mathrm{~N}_{y}$ or $\mathrm{TiO}_{2}$ in the case of Al-capped TiN samples, that would lead to an enhanced intensity in the BE region from 457 to $459 \mathrm{eV}$. The corresponding $\mathrm{N}$ 1s spectrum exhibits no extra peak on the low $\mathrm{BE}$ side of the main line (cf. spectra from uncapped samples in Figs. 3(b)-3(d) and discussion below) proving that no $\mathrm{TiO}_{x} \mathrm{~N}_{y}$ is formed on the $\mathrm{Al}$-capped TiN surface. The presence of $\mathrm{TiO}_{2}$ species in Al-capped TiN films can be excluded based on the $\mathrm{O}$ 1s spectrum (shown in Ref. 30) which contains a single peak at $\sim 532.5 \mathrm{eV}$ due to Al-oxide, and no extra intensity at BE from 529.8 to $530.0 \mathrm{eV}$, where potential contribution due to $\mathrm{TiO}_{2}$ is expected (see $\mathrm{O}$ 1s spectra from uncapped films in Figs. 4(a)-4(c) and Table 1). Thus, we conclude that Ti 2p and N 1s spectra presented in Figs 2(a) and 3(a) are representative of native TiN surface, clean from $\mathrm{TiO}_{x} \mathrm{~N}_{y}$ and $\mathrm{TiO}_{2}$ species.

The Ti $2 \mathrm{p}$ spin-orbit split 2p $3 / 2$ and $2 \mathrm{p}_{1 / 2}$ components appear at 455.03 and $460.97 \mathrm{eV}$, respectively. In addition, the satellite features (TiN-sat) are also present shifted by $3.0 \mathrm{eV}$ 
towards higher BE with respect to the primary peaks. This is in agreement with previous XPS analyses of polycrystalline TiN layers grown in-situ in an XPS system. ${ }^{15,16}$ The origin of this feature is widely discussed in the literature and several interpretations have been proposed including $\mathrm{t}_{1 \mathrm{~g}} \rightarrow 2 \mathrm{t}_{2 \mathrm{~g}}$ intra-band transitions (shake-up events), ${ }^{14,17}$ decrease in the screening probability of the core-hole created during photoionization by Ti 3d electrons, ${ }^{13,15,19,20}$ and structural effects, ${ }^{18}$ and the presence of grain boundaries between $\mathrm{TiN}$ and $\mathrm{TiO}_{2}$ in oxidized TiN. $^{23}$

Satisfactory fit of the $\mathrm{Ti} 2 \mathrm{p}$ spectrum from native $\mathrm{TiN}$ surface requires asymmetric functions for the main components (see Fig. 2(a)), which can be explained by energy loses due to simultaneous excitations of valence electrons, as the density of states near and at the Fermi level is high. TiN-sat peaks are well-represented by Voigt functions with 95\% Lorentzian ingredient. The corresponding $\mathrm{N}$ 1s spectrum, shown in Fig. 3(a), is dominated by main peak centered at $397.34 \mathrm{eV}$ and assigned to TiN. The low-intensity feature ( $2 \%$ of the total $\mathrm{N} 1 \mathrm{~s}$ peak area) to the high $\mathrm{BE}$ side of the main peak, at $399.35 \mathrm{eV}$, is assigned to the satellite.

As emphasized above, the main ambition of the present study is to achieve a self-consistent model that not only satisfies qualitative agreement between component peaks (not the least TiN contributions present in both $\mathrm{Ti} 2 \mathrm{p}$, and $\mathrm{N}$ 1s spectra), but also fulfills the quantitative requirement (e.g., the area ratio between TiN component in $\mathrm{Ti} 2 \mathrm{p}$ and $\mathrm{N} 1 \mathrm{~s}$ spectra is 1:1 for a stoichiometric sample). Table 1 summarizes all fitting results including concentrations assigned to each of the individual components. The N/Ti ratio due to TiN contributions (including satellites) is 1.02 , in very good agreement to the bulk value of $1 \pm 0.01$ obtained from RBS.

\subsection{Air-exposed TiN films}

Input from native TiN Ti $2 \mathrm{p}$ and $\mathrm{N}$ 1s spectra deconvolution presented above is used to create peak models for a more complex case of a series of air-exposed TiN films. By varying 
the venting temperature after film growth TiN films are exposed to atmosphere at temperatures ranging from 29 to $430{ }^{\circ} \mathrm{C}$, which results in a gradual change in the surface composition as evident from a series of Ti 2p and N 1s spectra shown in Figs. 2(b)-(d) and 3(b)-(d). For completeness, peak models for corresponding O 1s (Fig. 4) and C 1s (Fig. 5) spectra are also included. The air exposure time is less than $10 \mathrm{~min}$, necessary to transfer the sample from the growth chamber into the XPS instrument, which is the situation commonly accounted in the everyday XPS practice as in situ XPS capability is rare. Hence, there is a great need for ways to extract chemical information in a non-destructive way, i.e., without involving noble gas ion etch.

Changes in the overall appearance of $\mathrm{Ti} 2 \mathrm{p}$ and $\mathrm{N}$ 1s spectra with respect to that of native TiN indicate the presence of new chemical species in the surface region and make the curve fitting task very challenging, or even hopeless, if no prior reference peak model exists. In order to extract reliable chemical information, we construct a peak model with input from native TiN (including the satellite peaks) to correctly represent the contribution from unaffected, deeperlying TiN regions in the Ti 2p and $\mathrm{N}$ 1s spectra, and add a minimum number of new component peaks necessary to obtain a high-quality fit. The line shape, $2 \mathrm{p}_{3 / 2}-2 \mathrm{p}_{1 / 2} \mathrm{BE}$ splitting, and the $2 \mathrm{p}_{3 / 2} / 2 \mathrm{p}_{1 / 2}$ area ratio for pairs of TiN and TiN-sat peaks are thus copied from the Ti $2 \mathrm{p}$ peak model of native TiN, while FWHM and peak intensities are allowed to vary. In addition, the BE difference between TiN and TiN-sat peaks and the relative TiN/TiN-sat peak areas are fixed at values determined by the modelling of the native TiN spectrum. Similarly, for all $\mathrm{N} 1 \mathrm{~s}$ spectra, the TiN and TiN-sat line shapes, the relative BE difference, as well as the TiN/TiN-sat peak areas are fixed at the values obtained from modelling of native TiN spectrum.

Contrary to common practice, the assignment of new spectral contributions is not based solely on the reference BE values, which differ greatly, as indicated in Fig. 1, preventing unambiguous and deterministic assignment of observed spectral features. We instead also seek 
to establish quantitative self-consistency between Ti 2p, N 1s, O 1s, and C 1s core-level spectra. Thus, for start, the peaks considered should be in the BE range corresponding to given chemical species.

The comparison of Ti 2p and $\mathrm{N}$ 1s spectra of air-exposed TiN films to that of native TiN surface clearly indicates that new chemical species are present at the surface. Ti 2p spectra gives a direct evidence for two new compounds. First, an additional spin-split doublet with the $\mathrm{Ti} 2 \mathrm{p}_{3 / 2}$ peak at the binding energy varying from $458.18 \mathrm{eV}$ with $T_{v}=29{ }^{\circ} \mathrm{C}$ to 458.60 with $T_{v}$ $=430{ }^{\circ} \mathrm{C}$ is assigned to the $\mathrm{TiO}_{2}$ formation. This particular assignment is only partly based on the reference BE values, which cover the range from 458.0 to $459.6 \mathrm{eV}$ (see Fig. 1), i.e., much wider than the presently measured BE spread. An additional, and stronger evidence for the $\mathrm{TiO}_{2}$ formation comes from the fact that the corresponding peak is present in the $\mathrm{O} 1 \mathrm{~s}$ spectra (Fig. 4(a)-(c)) at 529.9 $\pm 0.1 \mathrm{eV}$. BE of this peak agrees well with the earlier reports, see Ref.38 and references therein. More importantly, however, as indicated in Table 1, the peak area ratio between $\mathrm{TiO}_{2}$ contributions in the $\mathrm{Ti} 2 \mathrm{p}$ and $\mathrm{O}$ 1s spectra results in the elemental concentration ratio $\mathrm{O} / \mathrm{Ti}=2.00 \pm 0.03$, for all $T_{v}$ values, assuring not only qualitative, but also quantitative cross-peak self-consistency of the model. Such requirement of quantitative cross-peak selfconsistency, essentially never imposed in the XPS literature, highly increases reliability of the so-extracted chemical information.

The apparent change in the Ti 2p BE with increasing venting temperature is associated with an increasing thickness of the surface $\mathrm{TiO}_{2}$ layer, which eventually completely dominates the surface of the $T_{v}=430{ }^{\circ} \mathrm{C}$ TiN samples, as evident from the relative peak intensities in Figs. 2(d) and 3(d), as well as, results summarized in Tab.1. Thicker, non-conducting, oxide leads to the buildup of a positive charge at the surface (surface charging) causing peaks belonging to species located either within, or on top of the oxide layer to shift towards higher BE. 
$\mathrm{TiO}_{2}$ formation results in a release of molecular $\mathrm{N}_{2}$ according to the reaction $\mathrm{TiN}+\mathrm{O}_{2} \rightarrow \mathrm{TiO}_{2}+1 / 2 \mathrm{~N}_{2}$, which is particularly well reflected in the $\mathrm{N} 1$ s spectrum of the $T_{v}=$ $430{ }^{\circ} \mathrm{C}$ film indicating dramatic loss of $\mathrm{N}$ (see Tab.1) and a formation of new broad peak at $402.4 \mathrm{eV}$ due to interstitially incorporated molecular nitrogen. The latter feature has been observed previously after prolonged TiN anneal studies in the $\mathrm{O}_{2}$-containing atmospheres. ${ }^{21,39}$

Second compound that forms at the surface of TiN films upon air exposure gives rise to another pair of spin-split peaks with the Ti 2p $3 / 2$ component at $456.6 \pm 0.1 \mathrm{eV}$. This contribution is especially intense in the case of $T_{V}=29^{\circ} \mathrm{C}$ and $T_{V}=330{ }^{\circ} \mathrm{C}$ samples (Figs. 2(b)-(c)) and correlates very well to the new low-BE feature that appears in the corresponding $\mathrm{N} 1$ s spectra (Figs. 3(b)-(c)) at 396.0 \pm 0.1 . As indicated in Fig. 1, the possible options in the relevant Ti 2p BE range, include $\mathrm{TiO}_{x} \mathrm{~N}_{y}$ and $\mathrm{Ti}_{2} \mathrm{O}_{3}$. However, the observed $\mathrm{Ti} 2 \mathrm{p}-\mathrm{N}$ 1s cross-peak correlation strongly indicates that, in addition to oxygen, the new compound contains both $\mathrm{Ti}$ and $\mathrm{N}$, which excludes the $\mathrm{Ti}_{2} \mathrm{O}_{3}$ formation. The low BE component in the $\mathrm{N}$ 1s spectra of airexposed or air-annealed TiN films has been observed previously ${ }^{18,21,22}$ and assigned to oxynitride formation, which leads to an increased ionic character of the $\mathrm{Ti}-\mathrm{N}$ bond (hence lower $\mathrm{BE}$ ). Due to lower oxidation state, the $\mathrm{TiO}_{x} \mathrm{~N}_{y}$ component in the corresponding $\mathrm{O} 1 \mathrm{~s}$ spectra appears on the higher $\mathrm{BE}$ side of the $\mathrm{TiO}_{2}$ peak (overlapping with carbooxide peaks, as discussed below). It is also worth to note that, contrary to their $\mathrm{TiO}_{2}$ counterparts, the $\mathrm{TiO}_{x} \mathrm{~N}_{y}$ Ti 2p peaks do not exhibit shift towards higher BE with increasing $T_{v}$. This is due to the deeper location of these species, under the surface $\mathrm{TiO}_{2}$ layer, which makes them insensitive to the surface charging.

The N/Ti ratio calculated for TiN components (including satellites) is 1.01 and 1.02 for TiN samples vented at 29 and $330{ }^{\circ} \mathrm{C}$, in very good agreement to the bulk value of $1 \pm 0.01$ obtained from RBS, while N/Ti drops to $\sim 0.46$ with $T_{v}=430{ }^{\circ} \mathrm{C}$ due to severe $\mathrm{N}$ loss discussed above. 
A complete peak model requires presentation and discussion of main core-level signals for all elements detected at the surface. Hence, Figure 5(a)-(c) shows the set of C 1s spectra from air-exposed TiN surfaces as a function of venting temperature. Carbon is found at different chemical states depending on $T_{v}$. The strongest peak in all spectra is the $\mathrm{C}-\mathrm{C} / \mathrm{C}-\mathrm{H}$ component at the $\mathrm{BE}$ that varies from 284.04 to $284.68 \mathrm{eV}$ with $T_{v}$ increasing from 29 to $430{ }^{\circ} \mathrm{C}$. This variation in $\mathrm{BE}$ follows closely that observed for the $\mathrm{TiO}_{2}$ component in $\mathrm{Ti} 2 \mathrm{p}$ spectra and is attributed to charging resulting from the gradual buildup of surface oxide layer, as discussed above. The observation is particularly important to note as the C-C/C-H C1s line is often used for BE referencing, ${ }^{40}$ while spectra presented in Fig. 5 clearly prove that such procedure is not justified even within the same materials system. Aligning all core level signals to the $C$ 1s peak would result in the BE of Ti 2p peak assigned to TiN varying by $0.64 \mathrm{eV}$ as a function of $T_{v}$, which is not acceptable.

Of primarily interest for the self-consistent model construction are $C$ 1s peaks related to the C-O bonds, as they set requirement for the corresponding contributions in the $\mathrm{O} 1$ s spectra. There are two $\mathrm{C}$ 1s peaks common for all spectra, shifted by 1.6 and $4.6 \mathrm{eV}$ to the higher $\mathrm{BE}$ side of the main $\mathrm{C}-\mathrm{C} / \mathrm{C}-\mathrm{H}$ peak. The magnitude of the chemical shifts strongly indicates that the first peak is due to the $\mathrm{C}-\mathrm{O}$ bond, while the second one is assigned to $\mathrm{O}=\mathrm{C}-\mathrm{O} \cdot{ }^{41,42}$ Thus, in addition to $\mathrm{TiO}_{2}$ and $\mathrm{TiO}_{x} \mathrm{~N}_{y}$, O 1s spectra is also expected to contain contributions from $\mathrm{CO}$ and $\mathrm{CO}_{2}$ species. Since little is known about the relative $\mathrm{BE}$ of these peaks, we chose to represent them in our model with one broader component (accounting also for $\mathrm{TiO}_{x} \mathrm{~N}_{y}$ ) present in all spectra at the $\mathrm{BE}$ of $\sim 531 \mathrm{eV}$.

Using atomic concentrations summarized in Tab.1 the oxynitride stoichiometry can be easily determined as $\mathrm{TiO}_{0.3} \mathrm{~N}_{0.7}$ for the $T_{v}=29{ }^{\circ} \mathrm{C}$ sample that changes to $\mathrm{TiO}_{0.4} \mathrm{~N}_{1.6}$ and $\mathrm{TiO}_{1.2} \mathrm{~N}_{0.8}$ with $T_{v}$ increasing to 330 and $430{ }^{\circ} \mathrm{C}$. The relative concentration ratio between 
oxynitride and $\mathrm{TiO}_{2}$ contributions increases with venting temperature from 0.6 with $T_{v}=29{ }^{\circ} \mathrm{C}$ to 1.0 in the case of $T_{v}=330{ }^{\circ} \mathrm{C}$ film and eventually 7.2 with the highest $T_{v}=430{ }^{\circ} \mathrm{C}$.

There are also a few contributions in the $\mathrm{C}$ 1s spectra that are of a minor importance for the application of the proposed model, nevertheless require a comment. First, the lowest BE component, here at 281.95 to $282.09 \mathrm{eV}$, is due to TiC species formed during film growth. ${ }^{43}$ The very low intensity of this contribution, $0.2-0.6$ at\%, justifies the fact that the corresponding component is omitted in the Ti $2 p$ spectra, where it would have accounted for less than $2 \%$ of the total $\mathrm{Ti} 2 \mathrm{p}$ area. It is worth to note that the $\mathrm{BE}$ of $\mathrm{Ti}-\mathrm{C}$ component is not affected by charging, since these species are located below the surface oxide layer.

The C 1s spectrum from the $T_{v}=29^{\circ} \mathrm{C}$ film cannot be fitted without an additional peak at $284.87 \mathrm{eV}$, which here is assigned to the contamination due to prolonged exposure to residual gases in high vacuum chamber necessary for the system to reach nearly-RT $T_{v}$. Within the group of minor contributions with no impact on the presented case is also the highest $\mathrm{BE} \mathrm{O} 1 \mathrm{~s}$ peak at 532.83-532.89 eV, best visible in the $T_{v}=29^{\circ} \mathrm{C}$ spectrum, and assigned to $\mathrm{H}_{2} \mathrm{O} .{ }^{24}$

\section{Conclusions}

We present a first self-consistent peak model developed to analyze XPS spectra of compound-forming materials, as demonstrated here for a set of TiN thin films grown by dc magnetron sputtering and oxidized to a different extent by varying the venting temperature $T_{V}$ after growth. Presented results constitute a model case for typical XPS application studies, wherein certain degree of atmosphere exposure during sample transfer to the XPS instrument is unavoidable and the challenge is to extract information about surface chemistry without imparting destructive ion etch prior to analyses. To enhance reliability of the Ti 2p, $\mathrm{N} 1$ s, $\mathrm{O} 1 \mathrm{~s}$, and C 1s XPS spectra deconvolution the whole process of model construction is not only guided by the comparison to the reference binding energy values that exhibit large spread making the peak assignment ambiguous, but also an additional constraint is imposed across all core-level 
spectra that requires both qualitative and quantitative self-consistency between component peaks belonging to the same chemical species. For example, the presence of $\mathrm{TiO}_{2}$ species on air-exposed TiN surface implies not only that $\mathrm{Ti} 2 \mathrm{p}$ and $\mathrm{O}$ 1s spectra contain $\mathrm{TiO}_{2}$ peaks at $\mathrm{BE}$ that falls within the previously reported range, but more importantly that the peak area ratio between $\mathrm{TiO}_{2}$ contributions in the $\mathrm{Ti} 2 \mathrm{p}$ and $\mathrm{O}$ 1s spectra is in good agreement with the compound stoichiometry. We advocate that such cross-peak self-consistency should be a prerequisite for reliable XPS peak modelling as it is both deterministic and provides enhanced credibility of obtained chemical information, instead of relying only on reference binding energy values with corresponding large ambiguity for a given material.

\section{Acknowledgements}

We acknowledge support from the VINN Excellence Center Functional Nanoscale Materials (FunMat) Grant 2005-02666, the Swedish Government Strategic Research Area in Materials Science on Functional Materials at Linköping University (SFO-Mat-LiU 2009-00971), and the Knut and Alice Wallenberg Foundation Scholar Grant 2011.0143. We thank Dr. Jens Jensen for help with ToF-E ERDA and RBS measurements. 


\section{Table caption}

Line shapes, binding energies, and atomic concentrations (in parentheses) derived from peak models presented in Figures 2-5.

\section{Figure captions}

Fig. 1. Summary of reported Ti 2p3/2 binding energy values for chemical states of Ti relevant to this work.

Fig. 2. Ti 2p XPS spectra obtained from air-exposed polycrystalline TiN films grown at 430 ${ }^{\circ} \mathrm{C}$ on $\mathrm{Si}(001)$ substrates and (a) capped in situ with 1.5-nm-thick Al layer, (b)-(d) uncapped and exposed to atmosphere at different venting temperatures ranging from 29 to $430{ }^{\circ} \mathrm{C}$.

Fig. 3. N 1s XPS spectra obtained from air-exposed polycrystalline TiN films grown at $430{ }^{\circ} \mathrm{C}$ on $\mathrm{Si}(001)$ substrates and (a) capped in situ with 1.5-nm-thick Al layer, (b)-(d) uncapped and exposed to atmosphere at different venting temperatures ranging from 29 to $430{ }^{\circ} \mathrm{C}$.

Fig. 4. O 1s XPS spectra obtained from air-exposed polycrystalline TiN films grown at $430{ }^{\circ} \mathrm{C}$ on $\mathrm{Si}(001)$ substrates and exposed to atmosphere at different venting temperatures (a) $29{ }^{\circ} \mathrm{C}$, (b) $330^{\circ} \mathrm{C}$, and (c) $430{ }^{\circ} \mathrm{C}$.

Fig. 5. C 1s XPS spectra obtained from air-exposed polycrystalline TiN films grown at $430{ }^{\circ} \mathrm{C}$ on $\mathrm{Si}(001)$ substrates and exposed to atmosphere at different venting temperatures (a) $29{ }^{\circ} \mathrm{C}$, (b) $330^{\circ} \mathrm{C}$, and (c) $430{ }^{\circ} \mathrm{C}$. 


\section{References}

${ }^{1}$ D. Gall, I. Petrov, J.E. Greene, J. Appl. Phys. 89 (2001) 401

${ }^{2}$ A.B. Mei, A. Rockett, L. Hultman, I. Petrov and J.E. Greene, J. Appl. Phys. 114 (2013) 193708

${ }^{3}$ D. McIntyre, J.E. Greene, G. Hakansson, J.-E. Sundgren, and W.-D. Münz, J. Appl. Phys. 67 (1990) 1542

${ }^{4}$ A. Ingason, F. Magnus, J.S. Agustsson, S. Olafsson, J.T. Gudmundsson, Thin Solid Films 517 (2009) 6731

${ }^{5}$ P. H. Mayrhofer, C. Mitterer, L. Hultman, H. Clemens. Progress in Materials Science 51 (2006) 1032

${ }^{6}$ H. Ljungcrantz, M. Odén, L. Hultman, J.E. Greene, J.-E. Sundgren, J. Appl. Phys. 80 (1996) 6725

${ }^{7}$ O. Knotek, M. Böhmer, T. Leyendecker, J. Vac. Sci. Technol. A 4 (1986) 2695

${ }^{8}$ T. Leyendecker, O. Lemmer, S. Esser, J. Ebberink, Surf. Coat. Technol. 48 (1991) 175

${ }^{9}$ J.M. Molarius, A.S. Korhonen, E. Harju, R. Lappalainen, Surf. Coat. Technol. 33 (1987) 117

${ }^{10}$ V.R. Parameswaran, J.-P. Immarigeon, D. Nagy, Surf. Coat. Technol. 52 (1992) 251

${ }^{11}$ I. Petrov, E. Mojab, F. Adibi, J.E. Greene, L. Hultman, J.-E. Sundgren, J. Vac. Sci. Technol. A 11 (1993) 11

12 J.S. Chun, P. Desjardins, C. Lavoie, C.-S. Shin, C. Cabral Jr., I. Petrov, J.E. Greene, J. Appl. Phys. 89 (2001) 7841

${ }^{13}$ L. Porte, L. Roux, J. Hanus, Phys. Rev. B 28 (1983) 3214

${ }^{14}$ I. Strydom and S. Hofmann, J. Electron Spectrosc. Relat. Phenom. 56 (1991) 85

${ }^{15}$ A. Arranz, C. Palacio, Surf. Sci. 600, 2510 (2006).

${ }^{16}$ I. Bertoti, M. Mohai, J.L. Sullivan, S.O. Saied, Surface and Interface Analysis 21, 467 (1994).

${ }^{17}$ D. Jaeger, J. Patscheider, J. Electron Spectrosc. Relat. Phenom. 185 (2012) 523

${ }^{18}$ P. Prieto, R.E. Kirby, J. Vac. Sci. Technol. A 13 (1995) 2819

${ }^{19}$ J. Patscheider, N. Hellgren, R.T. Haasch, I. Petrov, J.E. Greene, Phys. Rev. B 83 (2011) 125124

${ }^{20}$ G. Greczynski, J. Jensen, J.E. Greene, I. Petrov, and L. Hultman, Surf. Sci. Spectra 21 (2014) 35

${ }^{21}$ F. Esaka, K. Furuya, H. Shimada, M. Imamura, N. Matsubayashi, H. Sato, A. Nishijima, A. Kawana, H. Ichimura, and T. Kikuchi, J. Vac. Sci. Technol. A 15 (1997) 2521

${ }^{22}$ I. Milošev, H.-H.Strehblow, B. Navinšek, M. Metikoš-Huković, Surf. Interf. Anal. 23 (1995) 529

${ }^{23}$ N.C. Saha, H.G. Tompkins, J. Appl. Phys. 72 (1992) 3072

${ }^{24}$ National Institute of Standards and Technology (NIST) X-ray Photoelectron Spectroscopy Database, Version 4.1 compiled by A.V. Naumkin, A. Kraut-Vass, S.W. Gaarenstroom, and C.J. Powell, http://srdata.nist.gov/xps/ accessed on 2016-04-07

${ }^{25}$ X. Feng, M. Sun, X. Ma, G. Tang, Appl. Surf. Sci. 257 (2011) 9904

${ }^{26}$ R.R. Mohanta, V.R.R. Medicherla, K.L. Mohanta, N, C. Nayak, S. Majumder,V. Solanki, S. Varma, K. Bapna, D.M. Phase, V. Sathe, Appl. Surf. Sci. 325 (2015) 185

${ }^{27}$ S. Oktay, Z. Kahraman, M. Urgen, K. Kazmanli, Appl. Surf. Sci. 328 (2015) 255

${ }^{28}$ D. Jaeger, J. Patscheider, J. Electron Spectrosc. Relat. Phenom. 185 (2012) 523

${ }^{29}$ G. Greczynski, S. Mráz, L. Hultman, J.M. Schneider, Appl. Phys. Lett. 108 (2016) 041603

${ }^{30}$ G. Greczynski, I. Petrov, J.E. Greene, and L. Hultman, J. Vac. Sci. Technol. A 33 (2015) 05E101-1

${ }^{31}$ J. Jensen, D. Martin, A. Surpi, T. Kubart, Nucl. Instr. Meth. B 268 (2010) 1893

${ }^{32}$ Kratos Analytical Ltd.: library filename: "casaXPS_KratosAxis-F1s.lib"

${ }^{33}$ D. A. Shirley, Physical Review B 5 (1972) 4709

${ }^{34}$ See N. Fairley "XPS Lineshapes and Curve Fitting" in "Surface Analysis by Auger and X-ray Photoelectron Spectroscopy” Ed. D. Briggs and J.T. Grant, IM Publications, Manchester, 2003, p.397.

${ }^{35}$ J. F. Ziegler, J. P, Biersack, U. Littmark, "The Stopping and Range of Ions in Solids," vol. 1 of series

"Stopping and Ranges of Ions in Matter," Pergamon Press, New York (1984).

${ }^{36}$ The electron mean free path in TiN $\lambda=2.2 \mathrm{~nm}$ is determined based upon a separate set of experiments in which TiN/ZrN/Si(001) samples with TiN thicknesses varying from 0 to $20 \mathrm{~nm}$ are analysed. $\lambda$ is estimated from the attenuation of the $\mathrm{Zr} 3 \mathrm{~d}$ core level signal $(\mathrm{BE}=180 \mathrm{eV})$ excited with $\mathrm{Al} \mathrm{K \alpha} \mathrm{x}$-rays $(h v=1486.6 \mathrm{eV})$, resulting in an electron kinetic energy $E_{e} \sim 1300 \mathrm{eV}$. $E_{e}$ is lower for Ti 2p and $\mathrm{N}$ 1s electrons (1030 and $1080 \mathrm{eV}$, respectively) and the corresponding mean free paths are somewhat shorter than $2.2 \mathrm{~nm}$, thus, estimates of the percentage contributions to $\mathrm{Ti} 2 \mathrm{p}$ and $\mathrm{N}$ 1s core-level XPS signals from the $\mathrm{Ar}^{+}$ion beam altered surface layer are lower limits.

${ }^{37}$ R.T. Haasch, T. -Y. Lee, D. Gall, J. E. Greene, and I. Petrov, Surface Science Spectra 7, 193 (2000)

${ }^{38}$ I. Bertoti, M. Mohai, J.L. Sullivan, S.O. Saied, Applied Surface Science 84 (1995) 357

${ }^{39}$ I. Milosev, H.-H. Strehblow, and B. Navinsek, Surf. Coat. Technol. 74-75 (1995) 897.

${ }^{40}$ T.L. Barr, S. Seal, J. Vac. Sci. Technol. A 13 (1995) 1239

${ }^{41}$ See Appendix E, in Surface Analysis by Auger and X-ray Photoelectron Spectroscopy, edited by D. Briggs and J. T. Grant (IM Publications, Manchester, 2003).

${ }^{42}$ G. Beamson and D. Briggs, High Resolution XPS of Organic Polymers: The Scienta ESCA 300 Database (Wiley, Chichester, 1992). 
${ }^{43}$ G. Greczynski, S. Mráz, L. Hultman, J.M. Schneider, Appl. Surf. Sci. (2016), DOI: 10.1016/j.apsusc.2016.05.129 


\begin{tabular}{|c|c|c|c|c|c|c|}
\hline $\begin{array}{c}\text { Core } \\
\text { level }\end{array}$ & component & Line shape & $\begin{array}{c}\text { native TiN } \\
\text { (Al-capped) }\end{array}$ & $\begin{array}{c}\mathrm{TiN}, \mathrm{T}_{\mathrm{v}}=29{ }^{\circ} \mathrm{C} \\
\text { (air-exposed) }\end{array}$ & $\begin{array}{c}\mathrm{TiN}, \mathrm{T}_{\mathrm{v}}=330^{\circ} \mathrm{C} \\
\text { (air-exposed) }\end{array}$ & $\begin{array}{c}\mathrm{TiN}, \mathrm{T}_{\mathrm{v}}=430^{\circ} \mathrm{C} \\
\text { (air-exposed) }\end{array}$ \\
\hline $\mathrm{Ti}_{2 \mathrm{p}_{3 / 2}}$ & $\mathrm{TiN}$ & $\mathrm{GL}(80) \mathrm{T}(0.6)$ & $455.03(35.6)^{*}$ & $455.03(17.0)^{*}$ & $455.03(10.1)^{*}$ & $455.03(3.5)^{*}$ \\
\hline $\mathrm{Ti}_{2} \mathrm{p}_{3 / 2}$ & $\mathrm{TiN}-\mathrm{sat}$ & $\mathrm{GL}(95)$ & $458.03(13.9)^{*}$ & $458.03(6.5)^{*}$ & $458.03(3.9)^{*}$ & $458.03(1.3)^{*}$ \\
\hline $\mathrm{Ti} 2 \mathrm{p}_{3 / 2}$ & $\mathrm{TiO}_{2}$ & $\mathrm{GL}(60)$ & - & $458.18(4.3)^{*}$ & $458.29(10.4)^{*}$ & $458.60(20.9)^{*}$ \\
\hline $\mathrm{Ti} 2 \mathrm{p}_{3 / 2}$ & $\mathrm{TiO}_{\mathrm{x}} \mathrm{N}_{\mathrm{y}}$ & $\mathrm{GL}(30)$ & - & $456.59(7.0)^{*}$ & $456.60(10.8)^{*}$ & $456.53(2.9)^{*}$ \\
\hline $\mathrm{N} 1 \mathrm{~s}$ & $\mathrm{TiN}$ & $\mathrm{GL}(90)$ & $397.34(41.0)$ & $397.23(19.4)$ & $397.18(11.8)$ & $396.62(1.8)$ \\
\hline $\mathrm{N} 1 \mathrm{~s}$ & $\mathrm{TiN}-\mathrm{sat}$ & $\mathrm{GL}(0)$ & $399.35(9.5)$ & $399.24(4.3)$ & $399.19(2.6)$ & $399.63(0.4)$ \\
\hline $\mathrm{N} 1 \mathrm{~s}$ & $\mathrm{TiO} \mathrm{N}_{\mathrm{y}}$ & $\mathrm{GL}(0)$ & - & $396.17(4.8)$ & $395.96(12.9)$ & $395.68(2.5)$ \\
\hline $\mathrm{N} 1 \mathrm{~s}$ & $\mathrm{~N}{ }_{2}$ & $\mathrm{GL}(0)$ & - & $401.80(0.3)$ & $401.63(0.5)$ & $401.90(1.9)$ \\
\hline $\mathrm{O} 1 \mathrm{~s}$ & $\mathrm{TiO}$ & - & $529.81(8.5)$ & $529.82(21.1)$ & $529.98(41.4)$ \\
\hline $\mathrm{O} 1 \mathrm{~s}$ & $\mathrm{C}-\mathrm{O} / \mathrm{O}=\mathrm{C}-\mathrm{O}, \mathrm{TiO} \mathrm{N}_{\mathrm{y}}$ & $\mathrm{GL}(0)$ & - & $530.99(6.1)$ & $531.01(8.1)$ & $531.40(7.8)$ \\
\hline $\mathrm{O} 1 \mathrm{~s}$ & $\mathrm{H}{ }_{2} \mathrm{O}$ & $\mathrm{GL}(0)$ & - & $532.89(1.6)$ & $532.83(0.6)$ & - \\
\hline $\mathrm{C} 1 \mathrm{~s}$ & $\mathrm{TiC}$ & $\mathrm{GL}(0)$ & - & $282.09(0.6)$ & $281.95(0.2)$ & - \\
\hline $\mathrm{C} 1 \mathrm{~s}$ & $\mathrm{C}-\mathrm{C} / \mathrm{C}-\mathrm{H}$ & $\mathrm{GL}(0)$ & - & $284.04(18.3)$ & $284.10(4.1)$ & $284.68(13.9)$ \\
\hline $\mathrm{C} 1 \mathrm{~s}$ & $\mathrm{C}-\mathrm{O}$ & $\mathrm{GL}(0)$ & - & $285.67(2.2)$ & $285.74(2.1)$ & $286.24(2.3)$ \\
\hline $\mathrm{C} 1 \mathrm{~s}$ & $\mathrm{O}=\mathrm{C}-\mathrm{O}$ & $\mathrm{GL}(0)$ & - & $288.65(0.9)$ & $288.64(0.8)$ & $288.63(1.0)$ \\
\hline
\end{tabular}

* - both $\mathrm{Ti} 2 \mathrm{p}_{3 / 2}$ and $\mathrm{Ti} 2 \mathrm{p}_{1 / 2}$ included in quantification 


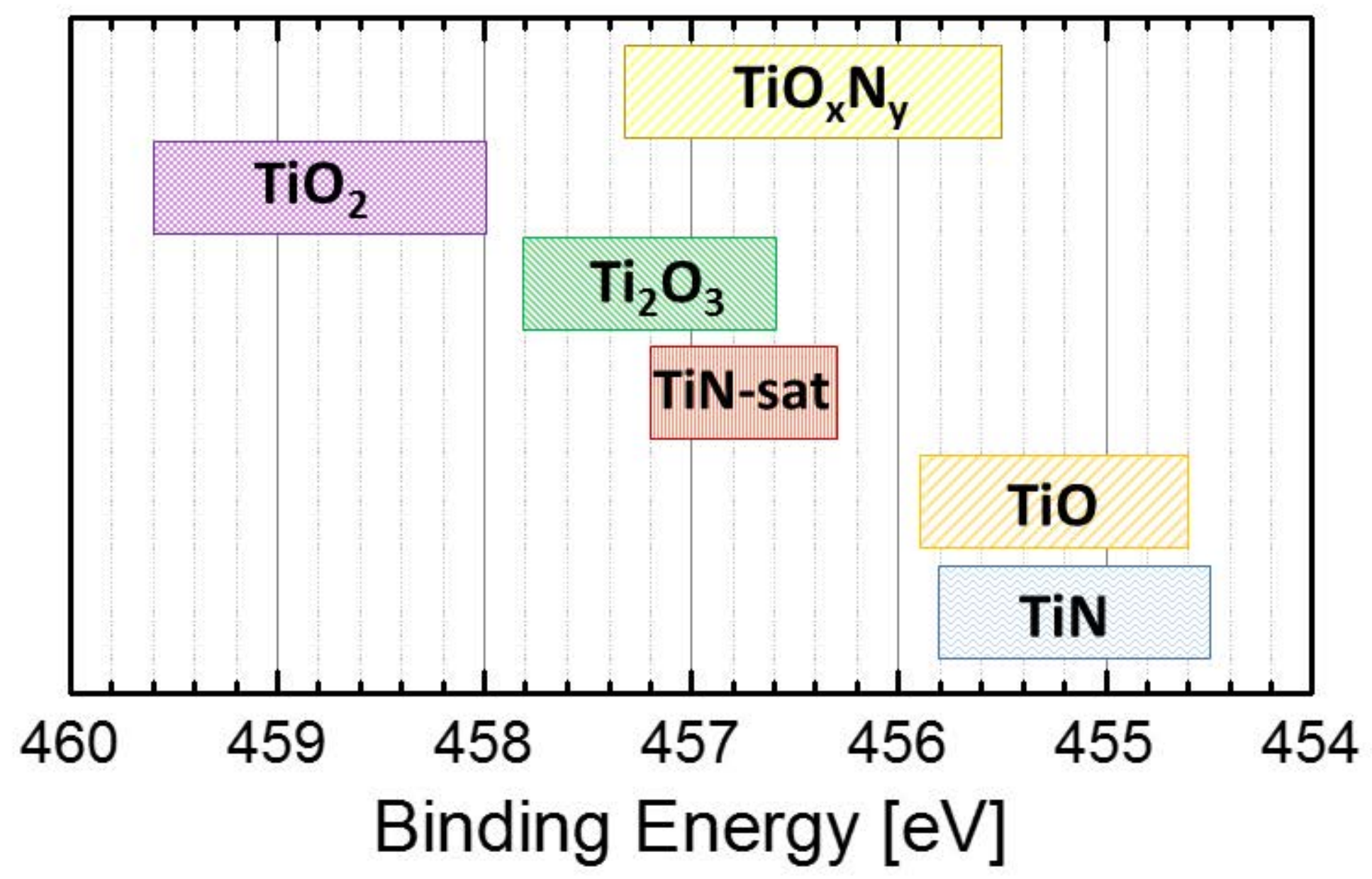

Fig. 1 


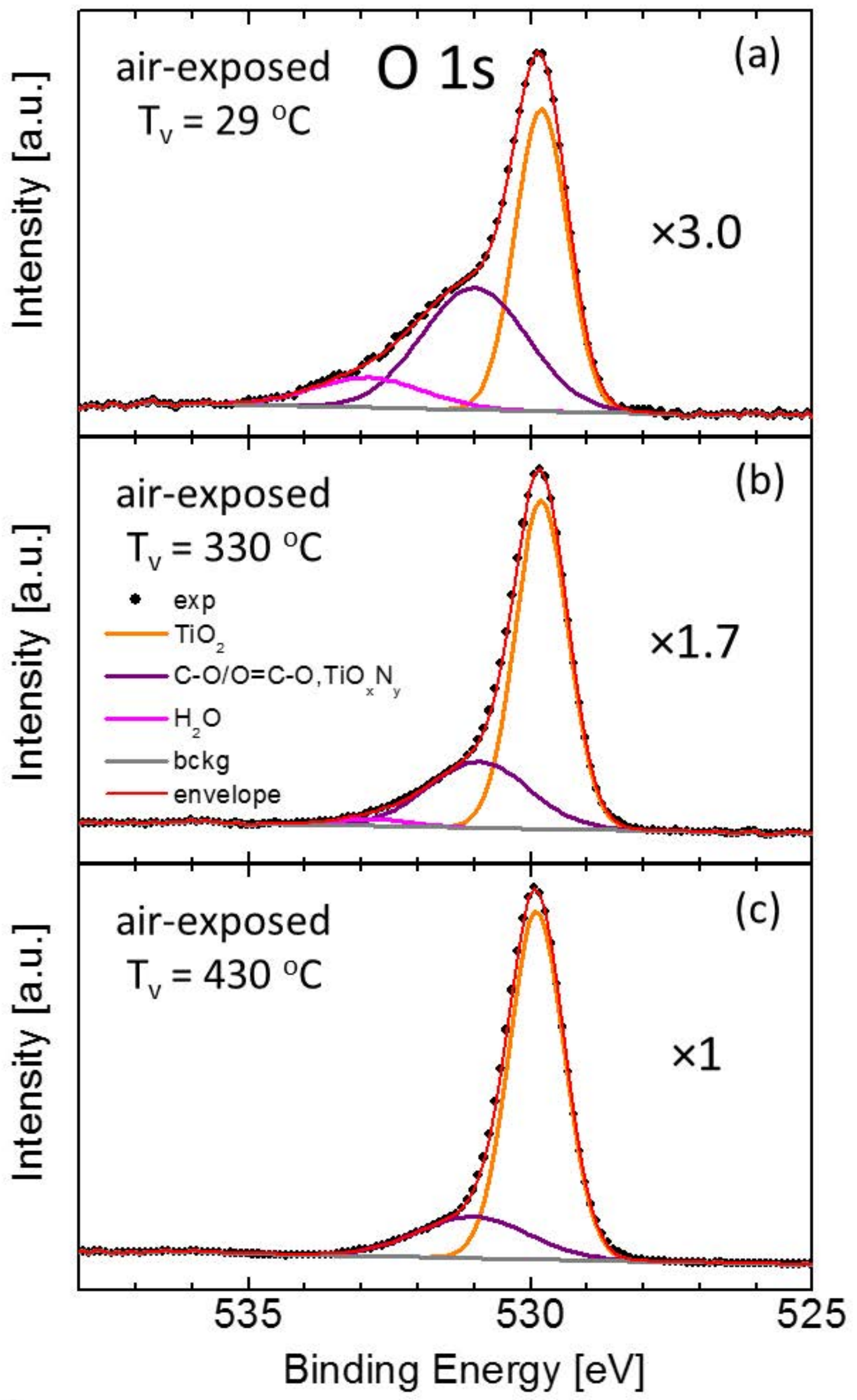

Fig. 4 


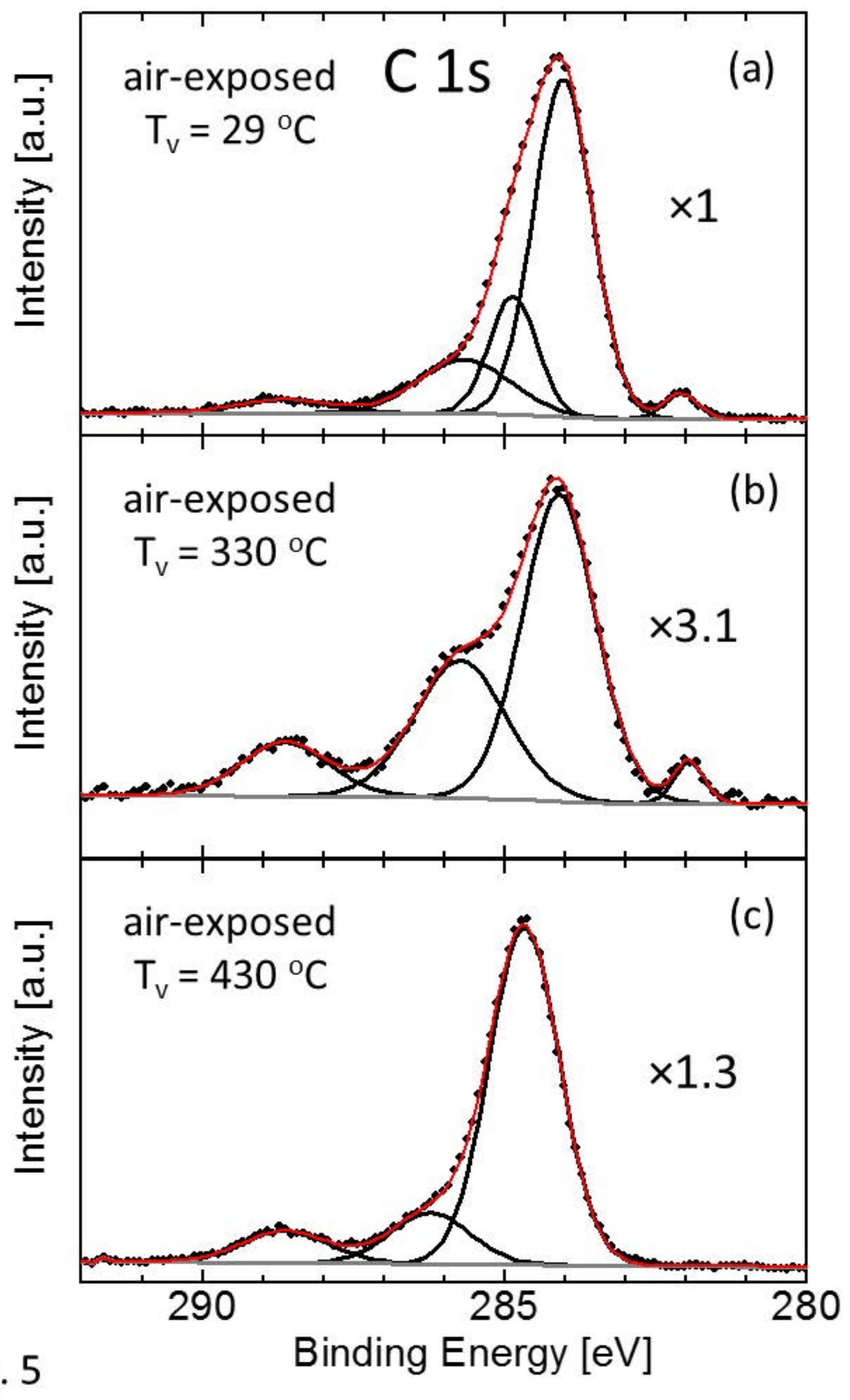

Fig. 5 